\title{
Economic change and graft outcome in kidney transplant recipients: a nationwide study of Korea
}

\author{
Sehoon Park , Jina Park ${ }^{1}$ Eun-Jeong Kang ${ }^{2}$, Yaerim Kim³ ${ }^{3}$ Yong Chul Kim¹, Yon Su Kim¹, Minsu Park ${ }^{4}$, Hajeong Lee
}

1Department of Internal Medicine-Nephrology, Seoul National University Hospital, Seoul, Korea
${ }^{2}$ Department of Internal Medicine-Nephrology, Ewha Womans University Seoul Hospital, Seoul, Korea
${ }^{3}$ Department of Internal Medicine-Nephrology, Keimyung University School of Medicine, Daegu, Korea
${ }^{4}$ Department of Biostatistics, Chungnam National University, Daejeon, Korea

Background: The socioeconomic status of kidney transplant (KT) recipients is closely associated with graft prognosis. Further study is warranted to investigate whether changes in socioeconomic status occur after KT alongside related differences in graft outcome.

Methods: We performed a nationwide observational cohort study reviewing the national claims database of Korea. Economic status was identified including income grades reflected in the insurance fee percentiles, employment status, and severe socioeconomic deprivation state. Changes in economic status in the period after KT was analyzed by linear regression. Graft failure outcome according to economic changes on 3 years after $\mathrm{KT}$ was also investigated.

Results: We included 18,487 KT recipients from 2002 to 2016 . The median age was 47 years old and $59 \%$ had male sex. The income percentile significantly decreased until 2 years after $\mathrm{KT}(\mathrm{P}<0.001)$ but there were no more significant changes from 2 years $(P=0.128)$. Employment decreased until 2 years after $K T(P<0.001)$; however, the employment rate significantly increased from 2 years to 5 years after $\mathrm{KT}(\mathrm{P}<0.001)$. Severe socioeconomic deprivation showed similar trends, as the proportion worsened until 2 years $(P<0.001)$ but relieved afterward $(P<0.001)$. Those employed before $K T$ but became unemployed 3 years after $K T$ showed a significantly higher risk of graft failure (adjusted hazard ratio [HR], $1.47[1.13,1.92] ; \mathrm{P}=0.004$ ) than those who remained employed. On the other hand, those with severe economic deprivation before $\mathrm{KT}$ but being improved economic status to non-aided showed better graft prognosis (adjusted HR, $0.50[0.34,0.74] ; \mathrm{P}=0.001$ ) than those who remained in the aided people.

Conclusions: Economic status seemed to be changed dynamically after KT. Although economic status worsened in the acute period after $\mathrm{KT}$, the employment rate increased and the proportion of severe economic deprivation decreased in long term. Improved economic status and employment were associated with different graft outcomes in KT recipients.

\footnotetext{
(c) The Korean Society for Transplantation

This is an Open Access article distributed under the terms of the Creative Commons Attribution Non-Commercial License (http://creativecommons.org/licenses/by-nc/4.0/) which permits unrestricted non-commercial use, distribution, and reproduction in any medium, provided the original work is properly cited
} 